\title{
Biomarker-based diagnosis of cognitive disorders in a case series
}

\author{
Elisabeth Kapaki', Vasilios C. Constantinides ${ }^{1}$, Efstratios-Stylianos Pyrgelis ${ }^{1}$, Panagiotis G. Paraskevas ${ }^{2}$ \\ John D. Papatriantafyllou ${ }^{3}$, George P. Paraskevas ${ }^{1}$
}

\begin{abstract}
'Neurodegenerative Disorders and Neurochemistry and Biological Markers Units, 1st Department of Neurology, National and Kapodistrian University of Athens, School of Medicine, Eginition Hospital, Athens 11528, Greece.

2Department of Nursing, Technological Educational Institute of Crete, School of Health and Welfare Services, Heraklion, Stauromenos 71004, Crete, Greece.

${ }^{3}$ Third Age Day Care Center IASIS, Athens-Glyfada \& Memory Clinic, Medical Center Athens-Marousi, Maroussi, Athens 15125, Greece.
\end{abstract}

Correspondence to: Dr. Efstratios-Stylianos Pyrgelis, Neurodegenerative Disorders and Neurochemistry and Biological Markers Units, 1st Department of Neurology, National and Kapodistrian University of Athens, School of Medicine, Eginition Hospital, 72 Vas. Sophias Ave, Athens 11528, Greece. E-mail: stratospyrg@yahoo.gr

How to cite this article: Kapaki E, Constantinides VC, Pyrgelis ES, Paraskevas PG, Papatriantafyllou JD, Paraskevas GP. Biomarker-
based diagnosis of cognitive disorders in a case series. Neuroimmuno/ Neuroinflammation 2020;7:319-29. http://dx.doi.org/10.20517/2347-8659.2019.26

Received: 28 Dec 2019 First Decision: 8 Apr 2020 Revised: 7 May 2020 Accepted: 26 May 2020 Available online: 12 Jul 2020

Academic Editor: Athanassios P. Kyritsis Copy Editor: Cai-Hong Wang Production Editor: Jing Yu

\begin{abstract}
The classical cerebrospinal fluid biomarkers of Alzheimer's Disease (namely total tau, phospho-tau and amyloid beta peptide) have received much attention, since they can detect the biochemical fingerprint of Alzheimer's disease and serve as a diagnostic aid for correct diagnosis of cognitive disorders during life. In this case series, we present 6 examples of patients with cognitive impairment of various types and severities and how biomarker data were helpful in every day diagnostic approach, combined with clinical, neuropsychological and imaging data and based on the most recent guidelines and recommendations.
\end{abstract}

Keywords: Cerebrospinal fluid, tau, phospho-tau, amyloid-beta, Alzheimer's disease, frontotemporal dementia, vascular cognitive impairment

\section{INTRODUCTION}

Until relatively recently, Alzheimer's disease (AD) was diagnosed according to clinical criteria proposed more than 30 years ago, requiring the patient to be demented ${ }^{[1]}$. With time it became evident that

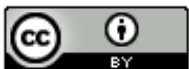

(C) The Author(s) 2020. Open Access This article is licensed under a Creative Commons Attribution 4.0 International License (https://creativecommons.org/licenses/by/4.0/), which permits unrestricted use sharing, adaptation, distribution and reproduction in any medium or format, for any purpose, even commercially, as long as you give appropriate credit to the original author(s) and the source, provide a link to the Creative Commons license, and indicate if changes were made.

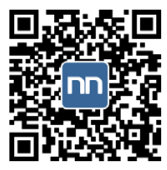


AD patients may present with mild cognitive impairment ${ }^{[2]}$, or may even be asymptomatic ${ }^{[3]}$. When symptomatic, amnestic dementia of the hippocampal type is the typical presentation ${ }^{[4,5]}$. However, atypical presentations are not infrequent, especially in presenile patients, including frontal-predominant, languagepredominant, "posterior" or mixed presentations ${ }^{[4,5]}$. Such presentations may lead to diagnostic confusion, whilst even the typical hippocampal amnestic presentation may occur in non-AD disorders ${ }^{[6]}$. Thus, clinical presentations or phenotypes are rather viewed as syndromes, and they are by no means synonymous with a specific disease. Various types of biomarkers may be helpful in the diagnostic approach of such typical or atypical presentations, and they have been incorporated in various diagnostic criteria for $\mathrm{AD}^{[2,4,5]}$.

Recently, the National Institute of Ageing and Alzheimer Association (NIA-AA) Research Framework group recommended a system for classifying subjects/patients on the basis of their biomarker profile, since it may result from different biomarker categories, especially neurochemical and imaging ${ }^{[7]}$. The objective was to update a scheme for defining and staging $\mathrm{AD}$ mainly across its entire spectrum, to be used for research purposes, either observational or interventional. A further shift in thinking is the separation of the syndrome from the disease, as symptoms are considered part of the disease continuum and not its definition. Looking towards a biological definition of $\mathrm{AD}$, as it is identified post mortem by accumulation of amyloid- $\beta$ and tau and reflected in vivo by biomarkers, the group discriminates them according to their molecular specificity [i.e., amyloid- $\beta(\mathrm{A} \beta)$ and pathological tau (phospho-tau)]. For this scope, they propose the AT(N) system ${ }^{[7]}$, where A stands for amyloid- $\beta$ plaques or associated pathological state, $\mathrm{T}$ for aggregated hyperphoshorylated tau or associated pathological state and $(\mathrm{N})$ for neurodegeneration. The parentheses are to indicate that it represents cumulative brain injury/neurodegeneration from all etiologies and is not specific for any certain etiology. A (C) component is used to define mental decline and staging, from cognitively unimpaired to mild cognitive impairment and finally dementia, according to cognitive symptoms and neuropsychological testing. Thus, each biomarker category can be dichotomized as positive $(+)$ or negative (-), resulting in eight different biomarker profiles and 3 "biomarker categories": normal $[\mathrm{A}-\mathrm{T}-(\mathrm{N})-]$, Alzheimer's continuum $[\mathrm{A}+\mathrm{T}-(\mathrm{N})-, \mathrm{A}+\mathrm{T}+(\mathrm{N})-, \mathrm{A}+\mathrm{T}+(\mathrm{N})+, \mathrm{A}+\mathrm{T}-(\mathrm{N})+]$ and suspected non-AD pathological change $[\mathrm{A}-\mathrm{T}+(\mathrm{N})-, \mathrm{A}-\mathrm{T}-(\mathrm{N})+, \mathrm{A}-\mathrm{T}+(\mathrm{N})+]^{[7]}$.

Here, we present a case series of six patients with different types of cognitive disorders using this system. Cases were selected with the only criterion being that they were educationally useful and interesting for clinicians and medical students, and all co-authors helped in the selection of cases, in an unblinded manner. We describe their clinical, imaging and cerebrospinal fluid (CSF) biomarker data and how these could be suggestive of diagnosis, according to the AT(N) system. All patients were analyzed routinely in our department as part of the everyday diagnostic approach and they were not included in any particular study.

Lumbar puncture was performed at 10-11 am, after overnight fasting, at the L4-L5 interspace, according to recently proposed recommendations on standardized operating procedures (SOPs) for CSF biomarkers ${ }^{[8]}$, as described elsewhere ${ }^{[9]}$. In brief, 4 polypropylene tubes were used for CSF collection. The initial tube $(2 \mathrm{~mL})$ was used for routine cytology and biochemistry and the 2 nd tube $(2 \mathrm{~mL})$ was used for determination of IgG index, oligoclonal bands and for syphilis serology. The last 2 tubes $(5 \mathrm{~mL}$ each) were immediately centrifuged, aliquoted in polypropylene tubes $\left(750 \mu \mathrm{L}\right.$ each) and, finally, stored at $-80{ }^{\circ} \mathrm{C}$ until analysis. Aliquots were thawed only once, just before analysis, which was performed within 6 months of storage.

CSF levels of total tau protein $\left(\tau_{\mathrm{T}}\right)$, amyloid- $\beta$ peptide and tau phosphorylated at threonine-181 $\left(\tau_{\mathrm{P}-181}\right)$ were measured blindly, in duplicate by double-sandwich, enzyme-linked immunosorbent assay (ELISA) using commercially available kits (Fujirebio, Gent, Belgium) according to the manufacturer's instructions, as previously described ${ }^{[9]}$. In-house standards were used during every to ensure minimal measurement error 
Table 1. Normal (cut-off) values of our laboratory ${ }^{[9]}$

\begin{tabular}{ll}
\hline CSF biomarker & Normal value \\
\hline total tau protein $\left(\tau_{\top}\right)$ & $<376 \mathrm{pg} / \mathrm{mL}$ \\
tau phosphorylated at threonine-181 $\left(\tau_{\mathrm{p}-181}\right)$ & $<57 \mathrm{pg} / \mathrm{mL}$ \\
amyloid- $\beta$ peptide with 42 amino acids $\left(A \beta_{42}\right)$ & $>682 \mathrm{pg} / \mathrm{mL}$ \\
$\mathrm{A} \beta_{42} / \mathrm{A} \beta_{40}$ & $>0.09$ \\
\hline
\end{tabular}

CSF: cerebrospinal fluid

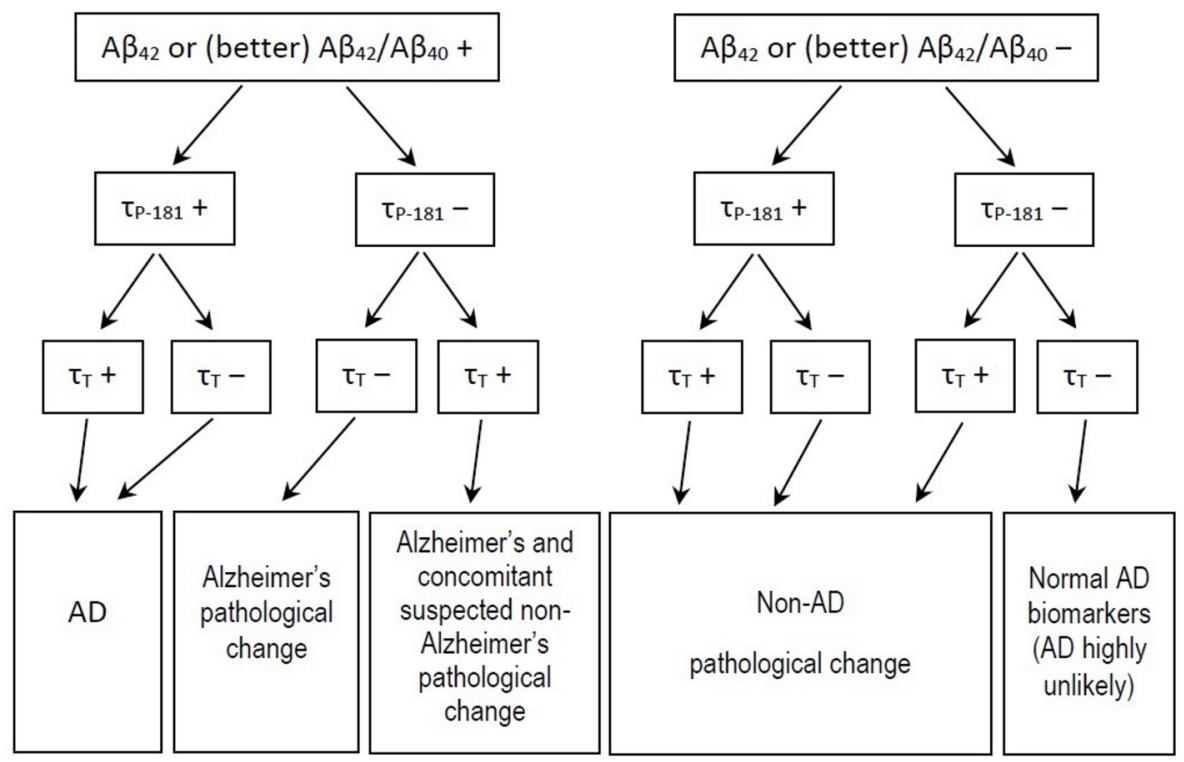

Figure 1. Flow chart of the use of cerebrospinal fluid biomarkers in clinical practice, according to the AT(N) system ${ }^{[7]}$. AD: Alzheimer's disease

$(\leq 3.3 \%)$, and inter-assay and intra-assay variations were $\leq 6.6 \%$ for all biomarker assays ${ }^{[10]}$. Cut-off values have been previously calculated by receiver operating curve (ROC) analysis ${ }^{[9,11}$. Table 1 shows the CSF biomarker categories used in our clinic/laboratory and their most recently used normal (cut-off) values ${ }^{[9]}$. Figure 1 presents a proposed simplified scheme for the diagnostic use of CSF biomarkers, according to the "philosophy" and nomenclature of the AT(N) system ${ }^{[7]}$.

\section{CASE REPORT}

\section{Case 1}

A 63-year-old female patient with no significant past medical history neither family history was admitted to the neurology department for gradually developed memory complaints over the last year with no impact on activities of daily living. Neuropsychological assessment revealed mild cognitive impairment with mini mental state examination (MMSE) ${ }^{[12]}$ score $27 / 30$ and frontal assessment battery $(\mathrm{FAB})^{[13]}$ score $16 / 18$. On magnetic resonance imaging (MRI) some degree of cortical atrophy in the parietal lobes was observed with relative preservation of the hippocampus [Figure 2]. Functional imaging study using single photon emission computerized tomography (SPECT) with ${ }^{99 \mathrm{~m}} \mathrm{Tc}-\mathrm{HMPAO}$ was normal. CSF biomarker analysis revealed increased $\tau_{\mathrm{T}}=545 \mathrm{pg} / \mathrm{mL}$ and $\tau_{\mathrm{P}-181}=81.8 \mathrm{pg} / \mathrm{mL}$ and decreased $\mathrm{A} \beta_{42}=480 \mathrm{pg} / \mathrm{ml}$ and $\mathrm{A} \beta_{42} /$ $\mathrm{A} \beta_{40}=0.059$. With all 3 biomarkers abnormal, the CSF profile was compatible with AD pathology and the patient was classified as $\mathrm{A}^{+} \mathrm{T}^{+}(\mathrm{N})^{+}$, suggesting "Alzheimer's disease with mild cognitive impairment" ${ }^{\text {" }}{ }^{7}$. During follow-up, she underwent two more neuropsychological assessments 4 and 8 years later, revealing progressive deterioration of cognition [Figure 3]. 

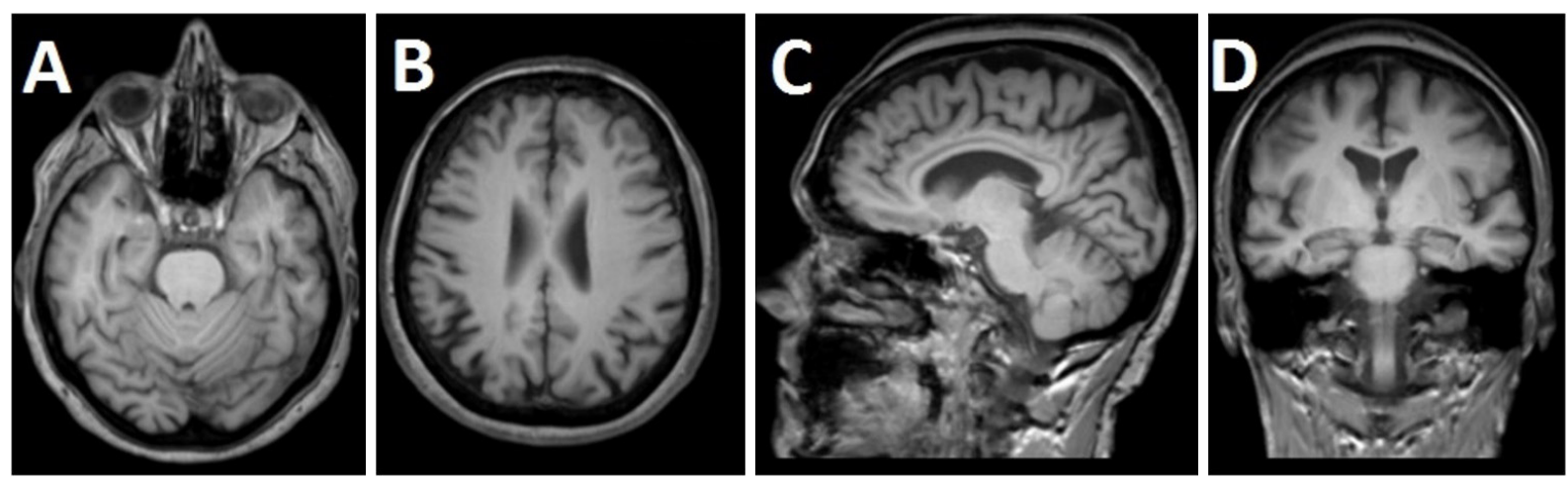

Figure 2. Brain magnetic resonance imaging of case 1 ( $T 1$ sequence), showing relative preservation of the hippocampus $(A, D)$ and some degree of parietal atrophy $(B, C)$

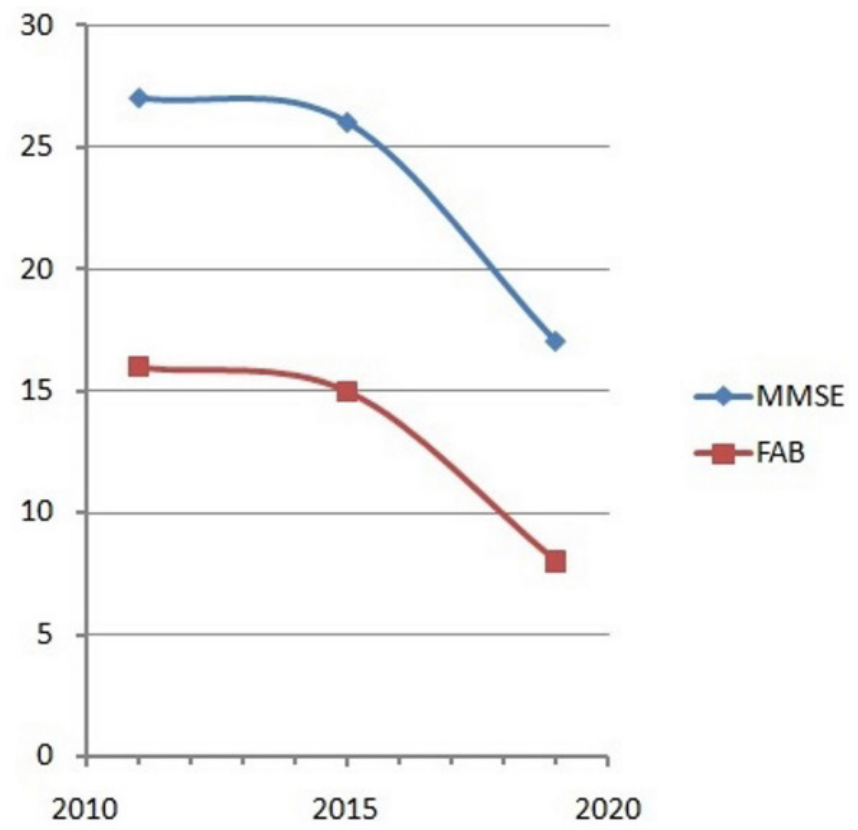

Figure 3. Progressive cognitive deterioration on mini mental state examination (MMSE) and frontal assessment battery (FAB) neuropsychological testing during the 8-year follow-up of case 1

\section{Case 2}

A 54-year-old female patient was referred for neurological evaluation due to progressive amnestic type dementia (MMSE: 21/30), with frontal and visuospatial components, evolving for approximately 5 years. MRI showed absence of atrophy. Biomarker analysis of CSF revealed normal $\tau_{\mathrm{T}}(261 \mathrm{pg} / \mathrm{mL})$, increased $\tau_{\mathrm{P}-181}$ (75 pg/mL), decreased $A \beta_{42}(168 \mathrm{pg} / \mathrm{mL})$ and decreased $A \beta_{42} / A \beta_{40}$ (0.04). The CSF profile was compatible with $\mathrm{AD}$, and according to the most recent recommendations, the patient was classified as $\mathrm{A}^{+} \mathrm{T}^{+}(\mathrm{N})$, suggesting "Alzheimer's disease with dementia" ${ }^{\text {"[7] }}$. In follow-up MRI, 3 and 4 years later, a progressive hippocampal and frontal-parietal atrophy was observed [Figure 4].

\section{Case 3}

A 71-year-old male patient was admitted to the neurology department due to dementia of mixed amnestic and frontal type. His brain MRI revealed ischemic lesion load but also frontal, perisylvian and frontoparietal atrophy more evident in the left hemisphere on axial fluid attenuated inversion recovery images, whereas according to T1 coronal images, the hippocampus was preserved [Figure 5]. Levels of 

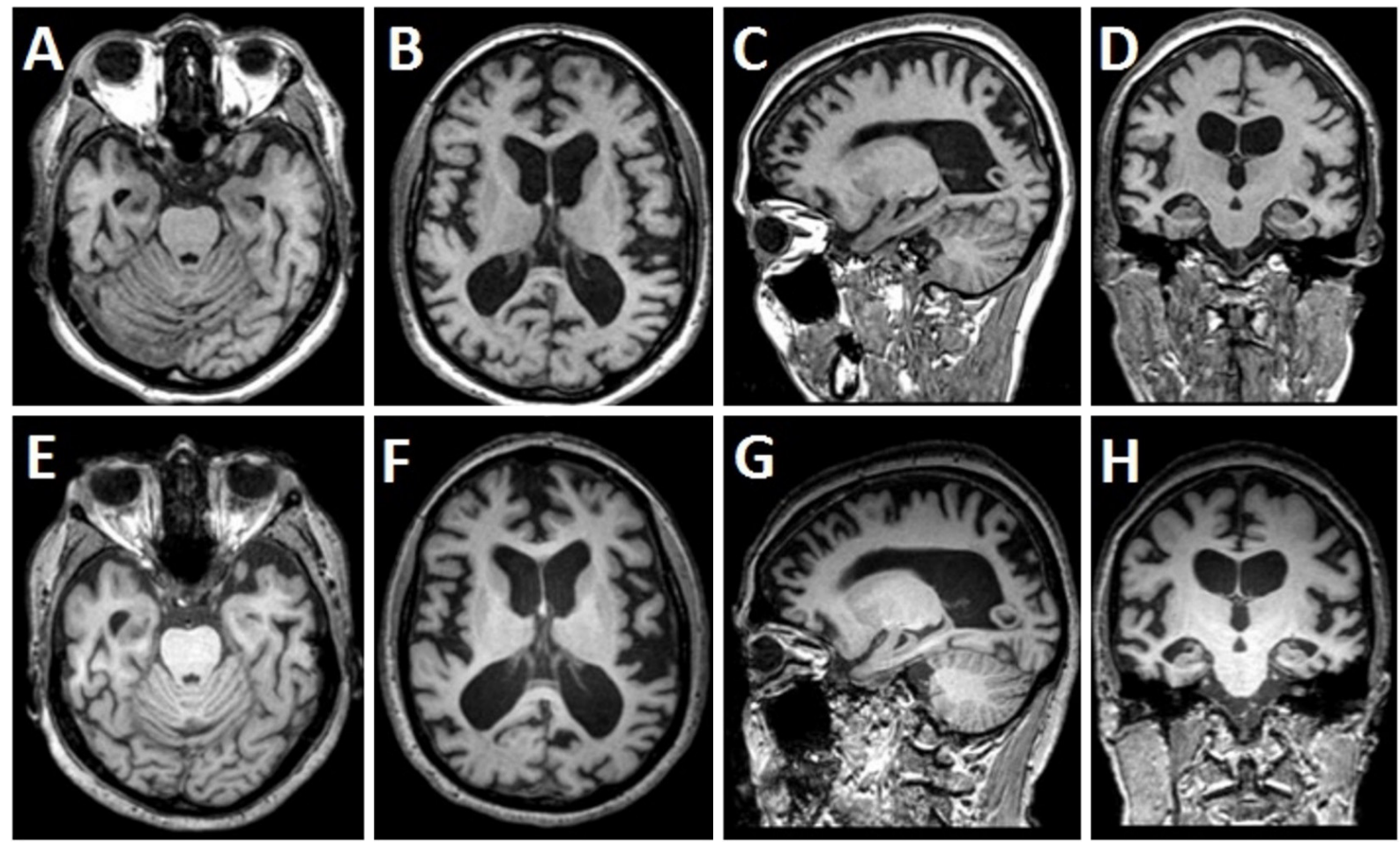

Figure 4. Brain magnetic resonance imaging of case 2 ( $T 1$ sequence) at age $57(A-D)$ and $58(E-H)$, showing progressive hippocampal atrophy $(A, D$ and $E, H)$, and frontal, perisylvian and parietal atrophy $(B, C$ and $F, G)$
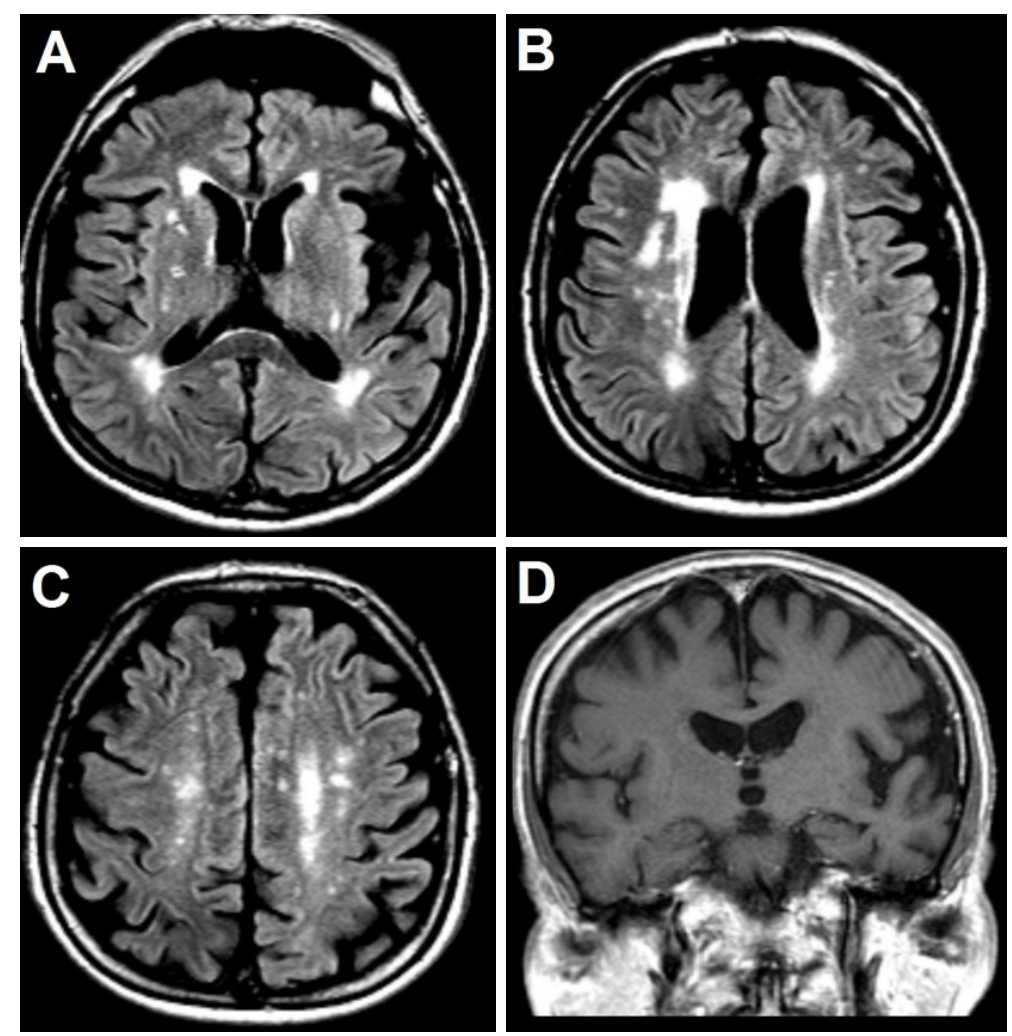

Figure 5. Brain magnetic resonance imaging of case 3. Fluid-attenuated inversion recovery images $(A, B, C)$ show ischemic lesion load and frontal, perisylvian and frontoparietal atrophy more evident in the left hemisphere. In coronal T1 section (D), the hippocampus is preserved 

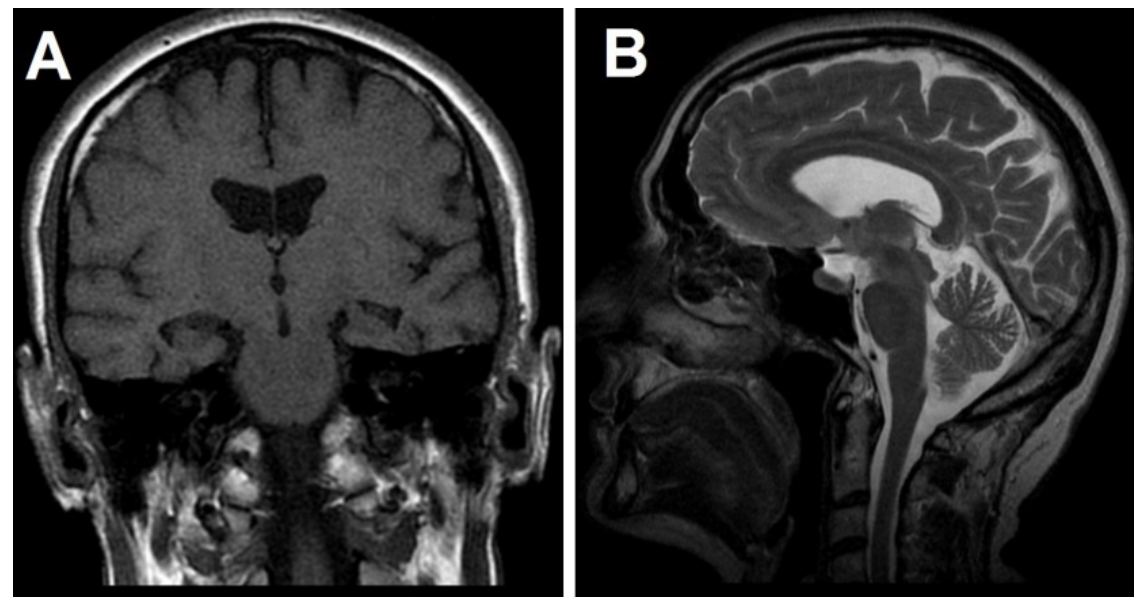

Figure 6. Brain magnetic resonance imaging of case 4. Coronal T1 section (A) reveals hippocampal atrophy. In sagittal T2 section (B), some degree of posterior frontal and parietal atrophy is observed

CSF biomarkers were: $\tau_{\mathrm{T}}=963 \mathrm{pg} / \mathrm{mL}, \mathrm{A} \beta_{42}=495 \mathrm{pg} / \mathrm{mL}, \tau_{\mathrm{P}-181}=87 \mathrm{pg} / \mathrm{mL}$ and $\mathrm{A} \beta_{42} / \mathrm{A} \beta_{40}=0.061$. With all 3 biomarkers abnormal, the CSF profile was compatible with $\mathrm{AD}$ pathology ${ }^{[7]}$ (in addition to subcortical small vessel disease).

\section{Case 4}

A 59-year-old female patient with typical amnestic dementia, fulfilling the clinical diagnostic criteria for probable $\mathrm{AD}^{[1]}$, was referred to the neurology department for evaluation. Hippocampal atrophy was observed on coronal T1 sequences and, additionally, some degree of posterior frontal and parietal atrophy on sagittal T2 sequences [Figure 6]. The CSF biomarker levels were: $\tau_{\mathrm{T}}=308 \mathrm{pg} / \mathrm{mL}, \mathrm{A} \beta_{42}=921 \mathrm{pg} / \mathrm{mL}$, $\tau_{\mathrm{P}-181}=36 \mathrm{pg} / \mathrm{mL}$ and $\mathrm{A} \beta_{42} / \mathrm{A} \beta_{40}=0.11$. Clinically, this "suspected non-Alzheimer disease pathophysiology" (SNAP) ${ }^{[14]}$ was otherwise compatible with an AD phenotype. However, with all 3 biomarker levels well within normal limits, the CSF profile was not compatible with $\mathrm{AD}^{[15]}$ and, according to the most recent recommendations, the patient was classified as $\mathrm{A}^{-} \mathrm{T}^{-}(\mathrm{N})^{+}$suggesting "non-Alzheimer's pathological change" ${ }^{\text {"[7] }}$.

\section{Case 5}

A 54-year-old female patient presented to our department with frontal-behavioral dementia, language disorder (mixed non-fluent and semantic components) and clinical and electrophysiological evidence of upper and lower motor neuron involvement. Her family history was positive for autosomal dominant dementia and/or ALS. On MRI T1 sequences, frontal and frontoparietal atrophy more evident to the left were present with relative preservation of the hippocampus [Figure 7]. Levels of CSF biomarkers were: $\tau_{\mathrm{T}}=$ $268 \mathrm{pg} / \mathrm{mL}, \mathrm{A} \beta_{42}=513 \mathrm{pg} / \mathrm{mL}, \tau_{\mathrm{P}-181}=20.4 \mathrm{pg} / \mathrm{mL}$ and $\mathrm{A} \beta_{42} / \mathrm{A} \beta_{40}=0.125$. Although the clinical presentation was suggestive of frontotemporal dementia (FTD)-amyotrophic lateral sclerosis (ALS), reduction of $A \beta_{42}$ was unexpected. However, correction for the total amyloid status revealed a normal $A \beta_{42} / A \beta_{40}$ ratio, excluding amyloid reduction ${ }^{[16,17]}$ and suggesting non-AD pathology. Given the clinical presentation, a TDP-43 proteinopathy was considered the most probable disorder. Indeed, genetic testing was positive for C9orf72 repeat expansion.

\section{Case 6}

A 40-year-old female with no past medical history was referred to the neurology department for presenile dementia. Neuropsychiatric symptoms began at the age of 34 and cognitive symptoms began three years later at the age of 37 and gradually deteriorated, fulfilling the clinical criteria for probable behavioral variant frontotemporal dementia ${ }^{[18]}$. MRI showed atrophy in the frontal and parietal lobes [Figure 8]. Levels 

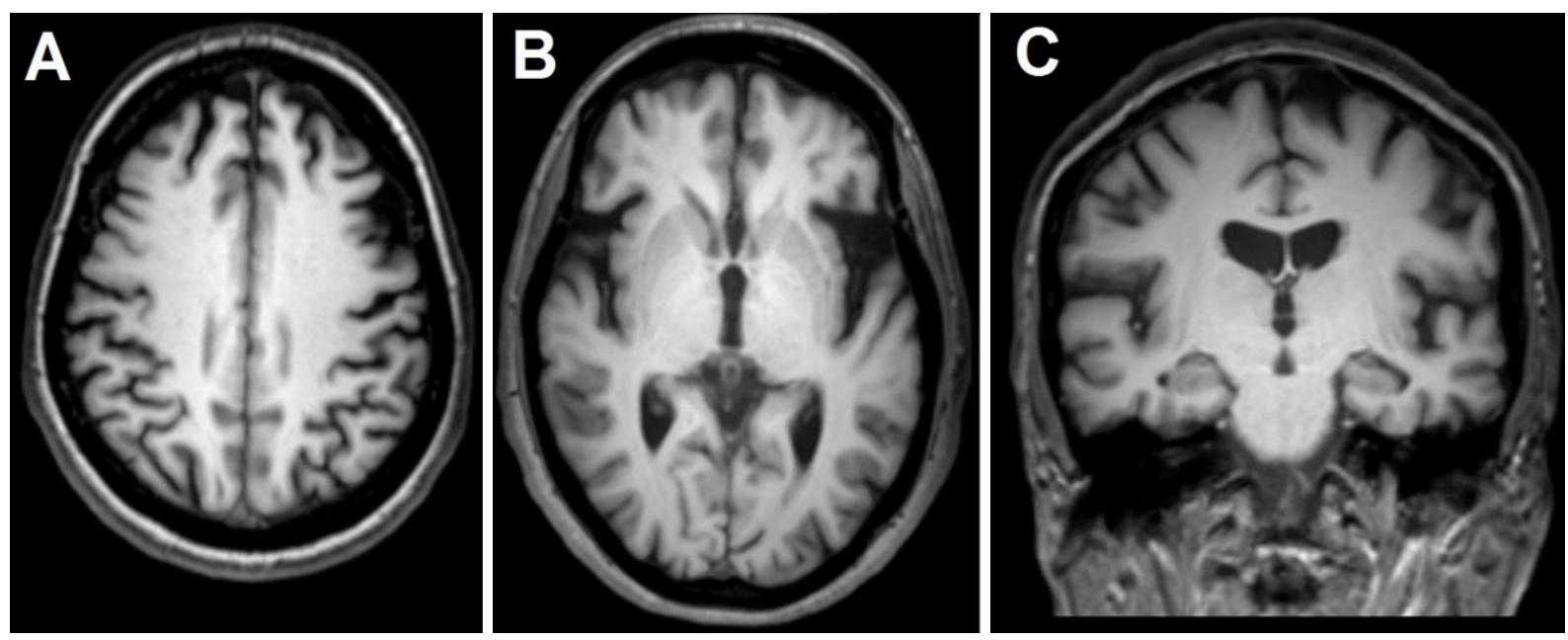

Figure 7. Brain magnetic resonance imaging ( $T 1$ sequence) of case 5 , showing frontal frontoparietal and sylvian atrophy more evident to the left $(A, B)$ with preservation of the hippocampus (C)
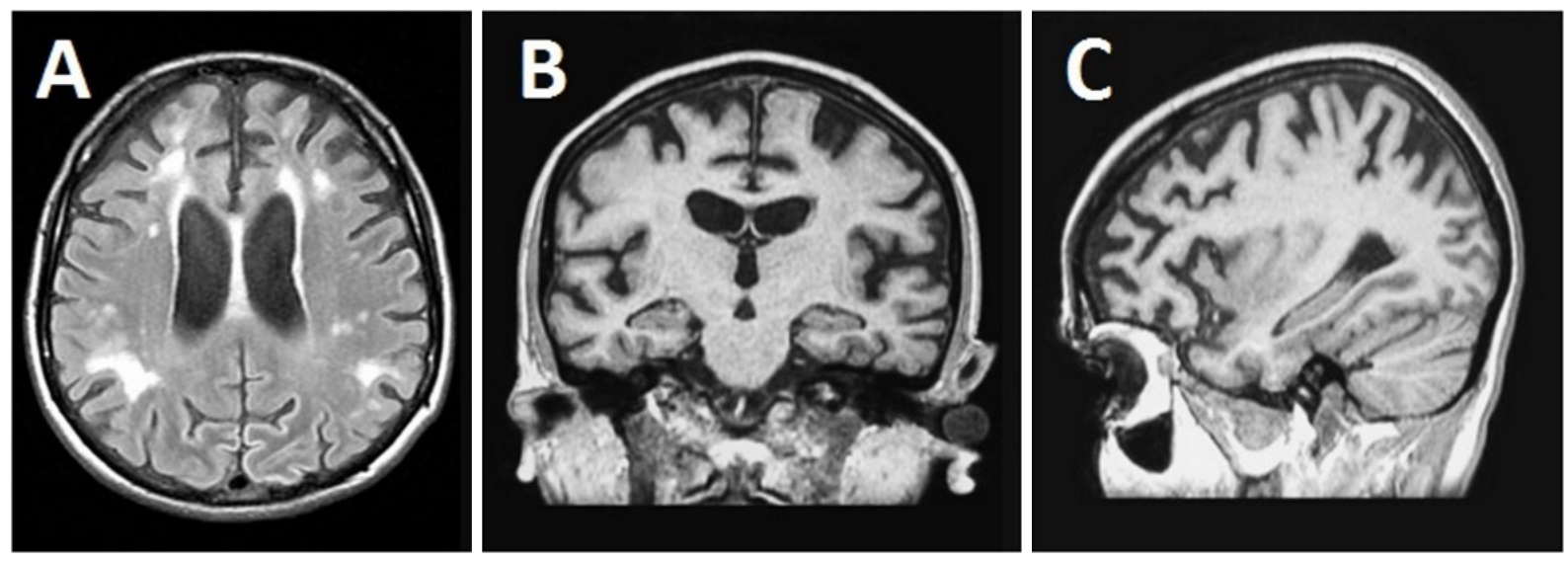

Figure 8. Brain magnetic resonance imaging of case 6. Axial fluid-attenuated inversion recovery image (A) showing white matter hyperintensities; coronal (B) and sagittal (C) T1 images reveal atrophy in frontal and parietal lobes (with some degree of left predominance) and preservation of the hippocampus

of CSF biomarkers were: $\tau_{\mathrm{T}}=1813 \mathrm{pg} / \mathrm{mL}, \mathrm{A} \beta_{42}=706 \mathrm{pg} / \mathrm{mL}, \tau_{\mathrm{P}-181}=67 \mathrm{pg} / \mathrm{mL}$ and $\mathrm{A} \beta_{42} / \mathrm{A} \beta_{40}=0.12$. With 2 biomarkers abnormal, the patient was classified as $\mathrm{A}^{-} \mathrm{T}^{+}(\mathrm{N})^{+}$, suggesting "non-Alzheimer's pathological change with dementia". Given the increased levels of $\tau_{\mathrm{P}-181}$, it was tempting to assume that frontotemporal dementia with tau pathology would be the most probable diagnosis ${ }^{[19]}$. Cerebral biopsy revealed severe tauopathy without accumulation of amyloid- $\beta$ or the presence of astrocytic plaques or tufted astrocytes. Genetic testing was negative for mutations in the MAPT and GRN genes.

\section{DISCUSSION}

We presented 6 patients as examples of a combined diagnostic approach based on clinical, imaging and CSF biomarkers, according to the $\mathrm{AT}(\mathrm{N})$ system $^{[7]}$. In case 1 , the diagnosis of $\mathrm{AD}$ was made in a symptomatic yet predementia stage (MCI). Case 2 was an amnestic dementia patient, and atypical features included presenile onset and absence of atrophy at presentation; however, CSF biomarkers revealed AD biochemistry and clinical-imaging progression was typical.

In case 3, a moderate ischemic lesion load could have contributed to the patient's symptoms, but $\mathrm{AD}$ was additionally present. This is a frequent scenario ${ }^{[20]}$, and CSF biomarkers are helpful in the discrimination between cases with pure vascular cognitive impairment and mixed cases (with additional AD) ${ }^{[21,22]}$. 
In case 4, a 59-year-old-female, clinically fulfilled the clinical criteria of an amnestic dementia of the AD type $^{[1]}$. However, clinical presentation does not always predict brain pathology. For example, AD can present with common amnestic dementia but also with a frontal behavioral-dysexecutive syndrome, the so-called "frontal variant of $\mathrm{AD}^{\text {" }}{ }^{[4,5]}$. Likewise, patients with FTLD pathologies may also present with an amnestic, AD-like syndrome. The term limbic-predominant age-related TDP-43 encephalopathy (LATE) has been recently introduced for at least some of these cases, and consensus-based recommendations and guidelines for diagnosis and staging have been formulated ${ }^{[6]}$. Thus, clinical, biochemical, neuropsychological and imaging data, all should be considered. Of course, there is always the possibility of false-negative or falsepositive results. Since all biomarkers become abnormal during prodromal stages of AD, all would be expected to be abnormal in a well-established AD dementia ${ }^{[23]}$. However, in this patient, all biomarkers were normal, dramatically reducing the possibility of false-negative results and pointing to a non-AD pathology. Indeed, with all CSF biomarkers normal, AD is considered highly unlikely according to recent recommendations ${ }^{[15]}$. Clinically the patient is not compatible with dementia with Lewy bodies (DLB), and to our knowledge, there are no robust, evidence-based data to support the use of standard AD treatments in non-AD, non-DLB patients. Thus, correct diagnosis would also avoid possibly unnecessary treatment(s) suitable for other diseases.

In case 5, TDP-43 proteinopathy was strongly considered from clinical presentation of combined phenotype FTD-ALS in the family, which is known to be related to a TDP-43 histopathology ${ }^{[24,25]}$. The CSF biomarker profile was compatible with non-AD pathology. In case 6, an AT(N) profile suggestive of non-AD pathology was also observed. However, in this patient, $\tau_{\mathrm{P}-181}$ was increased. Recently, it has been suggested that in an FTD-like patient, with no AD biomarker profile, increased $\tau_{\mathrm{P}-181}$ is more compatible with tau-pathology, while low $\tau_{\mathrm{P}-181}$ may be compatible with TDP-43 pathology ${ }^{\left[{ }^{19]}\right]}$. Thus, the tauopathy observed in brain biopsy was in accordance with this notion.

Soon after its publication, the AT(N) system triggered a lot of discussion and criticism. The concept of a disease viewed as a pathological/pathophysiological/biochemical entity unrelated to symptoms may not be easily accepted by some clinicians or the community ${ }^{[26]}$. However, given that the same disease may present with different clinical syndromes and that the same clinical presentation may be caused by different diseases, this new view is really a step forward, and this holds true not only for AD but also for many other neurodegenerative disorders. Furthermore, since the AD pathological process starts even decades prior to symptomatic onset, whilst CSF or imaging biomarkers become abnormal in the preclinical stage ${ }^{[23]}$, the need for adopting such a view/concept is further strengthened. However, many questions seek answers. For example, what about an $\mathrm{A}^{+} \mathrm{T}^{+} \mathrm{N}^{+}$patient with a clinical presentation suggestive of DLB. Is this due to mixed pathology (synucleinopathy and $\mathrm{AD})^{[5,27]}$ or due to $\mathrm{AD}$ with atypical presentation ${ }^{[28]}$ ? Another related question is a DLB-like patient with only amyloid biomarkers being positive. This is very common in DLB ${ }^{[29]}$. But, is this due to the synucleinopathy alone somehow triggering amyloid deposition unrelated to AD mechanisms, or are such patients "destined" to develop full-blown AD pathology if they live long enough? Furthermore, reduced $A \beta_{42}$ levels have been observed in some patients with pure vascular dementia ${ }^{[20]}$, including patients with inherited subcortical small vessel disease ${ }^{[30]}$, who do not have additional AD pathology, raising questions as to whether reduced $A \beta_{42}$ always suggests Alzheimer's pathological change.

Other CSF biomarkers may be of further help and improve the AT(N) system. Other forms of phosphotau such as $\tau_{\mathrm{P}-217}$ may perform better, compared to $\tau_{\mathrm{P}-181}{ }^{[31]}$. TDP-43 combined with $\tau_{\mathrm{T}}$ and $\tau_{\mathrm{P}-181}$ could enhance the diagnostic accuracy in the FTD spectrum ${ }^{[32,33]}$. CSF $\alpha$-synuclein levels could be useful in discriminating patients with $\mathrm{AD}$ from cognitively unimpaired subjects, patients with DLB and patients with Parkinson's disease dementia ${ }^{[34,35]}$. Blood-based biomarkers are quite promising as well, since classical AD biomarkers may also be measured in plasma. Plasma $\tau_{\mathrm{P}-181}$ could differentiate AD dementia from non-AD neurodegenerative diseases with accuracy similar to that of CSF $\tau_{\mathrm{P}-181}$ and tau-PET ${ }^{[36]}$, while plasma A $\beta_{42} /$ 
$\mathrm{A} \beta_{40}$ ratio has been associated with amyloid PET status in cognitively normal subjects ${ }^{[37]}$.

Inflammation biomarkers in CSF and blood have received much attention; however, whether they offer any added diagnostic value remains a matter of investigation. CSF $\alpha 1$-antichymotrypsin levels are increased both in vascular cognitive impairment (VCI) and clinically evident $\mathrm{AD}$, while elevated peripheral CRP levels may be associated with increased risk for VCI, but not $\mathrm{AD}^{[38]}$. Serum interleukin-15 levels have been found to be significantly lower in patients with $\mathrm{AD}$ in comparison to healthy subjects and patients with $\mathrm{VCI}^{[39]}$. On the other hand, CSF interleukin-15 levels are increased in AD and FTD, compared to patients with non-inflammatory neurological disorders ${ }^{[40]}$, while CSF interleukin-12 is reduced in AD, indicating altered inflammatory reactions ${ }^{[41]}$.

In neurodegenerative disorders, diagnosis should be established as soon as possible and preferably in a prodromal phase, before the onset of clinically significant dementia. Additionally, new emerging treatments or medications under investigation may be more effective when given in early stages. Therefore, timely and accurate diagnosis is mandatory to obtain potential benefits of novel treatments, but also for accurate inclusion of patients in clinical trials and for determining prognosis. As noted above in case 4, clinical phenotypes are not always tightly linked to the underlying pathology ${ }^{[5,6,9]}$ in contrast to biomarkers, some of which may have high molecular specificity. Nonetheless, CSF biomarkers are not a panacea, and their value should not be over-rated. They have disadvantages mainly due to the heterogeneity of research to date, but they still offer a very useful tool in early etiological diagnosis of neurodegenerative diseases, especially when combined with clinical and neuroimaging data ${ }^{[17]}$.

\section{DECLARATIONS}

\section{Authors' contributions}

Concept and definition of intellectual content: Kapaki E, Paraskevas GP

Clinical data acquisition and interpretation: Kapaki E, Constantinides VC, Pyrgelis ES, Paraskevas PG, Papatriantafyllou JD, Paraskevas GP

Biomarker determinations and interpretation of results: Kapaki E, Paraskevas GP

Manuscript preparation, editing and review: Kapaki E, Pyrgelis ES, Paraskevas PG, Papatriantafyllou JD, Paraskevas GP

\section{Availability of data and materials}

Not applicable.

\section{Financial support and sponsorship}

None.

\section{Conflicts of interest}

The authors declared that there are no conflicts of interest.

\section{Ethical approval and consent to participate}

Not applicable.

\section{Consent for publication}

All patients and/or relatives gave informed consent for publication of their clinical, biochemical and imaging data.

\section{Copyright}

(c) The Author(s) 2020. 


\section{REFERENCES}

1. McKhann G, Drachman D, Folsn M, Katzman R, Price D, et al. Clinical diagnosis of Alzheimer's disease: report of the NINCDSADRDA Work Group under the auspices of Department of Health and Human Services Task Force on Alzheimer's Disease. Neurology 1984;34:939-44.

2. Albert MS, DeKosky ST, Dickson D, Dubois B, Feldman HH, et al. The diagnosis of mild cognitive impairment due to Alzheimer's disease: recommendations from the National Institute on Aging-Alzheimer's Association workgroups on diagnostic guidelines for Alzheimer's disease. Alzheimers Dement 2011;7:270-79.

3. Sperling RA, Aisen PS, Beckett LA, Bennett DA, Craft S, et al. Toward defining the preclinical stages of Alzheimer's disease: recommendations from the National Institute on Aging-Alzheimer's Assocation workgroups on diagnostic guidelines for Alzheimer's disease. Alzheimers Dement 2011;7:280-92.

4. McKhann GM, Knopman DS, Chertkow H, Hyman BT, Jack CR Jr, et al. The diagnosis of dementia due to Alzheimer's disease: recommendations from the National Institute on Aging-Alzheimer's Association workgroups on diagnostic guidelines for Alzheimer's disease. Alzheimer's Dement 2011;7:263-69.

5. Dubois B, Feldman HH, Jacova C, Hampel H, Molinuevo JL, et al. Advancing research diagnostic criteria for Alzheimer's disease: the IWG-2 criteria. Lancet Neurol 2014;13:614-29.

6. Nelson PT, Dickson DW, Trojanowski JQ, Jack CR, Boyle PA, et al. Limbic-predominant age-related TDP-43 encephalopathy (LATE): consensus working group report. Brain 2019;142:1503-27.

7. Jack CR Jr, Bennett DA, Blennow K, Carrillo MC, Dunn B, et al. NIA-AA Research Framework: Toward a biological definition of Alzheimer's disease. Alzheimers Dement 2018;14:535-62.

8. del Campo M, Mollenhauer B, Bertolotto A, Engelborghs S, Hampel H. Recommendations to standardize preanalytical confounding factors in Alzheimer's and Parkinson's disease cerebrospinal fluid biomarkers: an update. Biomark Med 2012;6:419-30.

9. Paraskevas GP, Kaselimis D, Kourtidou E, Constantinides V, Bougea A, et al. Cerebrospinal fluid biomarkers as a diagnostic tool of the underlying pathology of primary progressive aphasia. J Alzheimers Dis 2017;55:1453-61.

10. Bourbouli M, Kapaki E, Petropoulou O, Paraskevas GP. Improved performance of CSF dementia biomarker measurements over time: the effect of quality control and harmonization programs. Biomark Applic 2018; doi: 10.29011/BMAP-126.100026.

11. Kapaki E, Paraskevas GP, Zalonis I, Zournas C. CSF tau protein and beta-amyloid (1-42) in Alzheimer's disease diagnosis: discrimination from normal ageing and other dementias in the Greek population. Eur J Neurol 2003;10:119-28.

12. Folstein M, Folstein S, McHugh PR. Mini-Mental State: a practical method for grading the cognitive state of patients for the clinician. J Psychiatr Res 1975;12:189-98.

13. Dubois B, Slachevsky A, Litvan I, Pillon B. The FAB: a frontal assessment battery at bedside. Neurology 2000;55:1621-26.

14. Jack CR Jr. PART and SNAP. Acta Neuropathol 2014;128:773-6.

15. Simonsen AH, Herukka SK, Andreasen N, Baldeiras I, Bjerke M, et al. Recommendations for CSF AD biomarkers in the diagnostic evaluation of dementia. Alzheimer's Dement 2017;13:274-84.

16. Lewczuk P, Lelental N, Spitzer P, Maler JM, Kornhuber J. Amyloid b 42/40 CSF concentration ratio in the diagnostics of Alzheimer's Disease: validation of two novel assays. J Alzheimer's Dis 2015;43:183-91.

17. Blennow K, Zetterberg H. The past and the future of Alzheimer's disease fluid biomarkers. J Alzheimers Dis 2018;62:1125-40.

18. Rascovsky K, Hodges JR, Knopman D, Mendez MF, Kramer JH, et al Sensitivity of revised diagnostic criteria for the behavioural variant of frontotemporal dementia. Brain 2011;134:2456-77.

19. Lleó A, Irwin DJ, Illán-Gala I, McMillan CT, Wolk DA, et al. A 2-Step cerebrospinal algorithm for the selection of frontotemporal lobar degeneration subtypes. JAMA Neurol 2018;75:738-45.

20. Wallin A, Nordlund A, Jonsson M, Blennow K, Zetterberg H, et al. Alzheimer's disease-subcortical vascular disease spectrum in a hospital-based setting: Overview of results from the Gothenburg MCI and dementia studies. J Cereb Blood Flow Metab 2016;36:95-113.

21. Paraskevas GP, Kapaki E, Papageorgiou SG, Kalfakis N, Andreadou E, et al. CSF biomarker profile and diagnostic value in vascular dementia. Eur J Neurol 2009;16:205-11.

22. Paraskevas GP, Constantinides VC, Pyrgelis ES, Kapaki E. Mixed Small Vessel Disease in a Patient with Dementia with Lewy Bodies. Brain Sci 2019;9. pii: E159

23. Jack CR Jr, Holtzman DM. Biomarker modeling of Alzheimer's disease. Neuron 2013;80:1347-58.

24. Josephs KA, Hodges JR, Snowden JS, Mackenzie IR, Neumann M, et al. Neuropathological background of phenotypical variability in frontotemporal dementia. Acta Neuropathol 2011;122:137-53.

25. Dong Y, Chen Y. The role of ubiquitinated TDP-43 in amyotrophic lateral sclerosis. Neuroimmunol Neuroinflammation 2018;5:5.

26. McCleery J, Flicker L, Richard E, Quinn TJ. When is Alzheimer's not dementia-Cochrane commentary on the national institute on ageing and Alzheimer's association research framework for Alzheimer's disease. Age Ageing 2019;48:174-7.

27. McKeith IG, Boeve BF, Dickson DW, Halliday G, Taylor JP, et al. Diagnosis and management of dementia with Lewy bodies: fourth consensus report of the DLB Consortium. Neurology 2017;89:88-100.

28. Skogseth R, Hortobágyi T, Soennesyn H, Chwiszczuk L, Ffytche D, et al. Accuracy of clinical diagnosis of dementia with lewy bodies versus neuropathology. J Alzheimer's Dis 2017;59:1139-52.

29. Paraskevas GP, Bougea A, Constantinides VC, Bourbouli M, Petropoulou O, et al. In vivo prevalence of alzheimer biomarkers in dementia with lewy bodies. Dement Geriatr Cogn Disord 2019;47:289-96.

30. Formichi P, Parnetti L, Radi E, Cevenini G, Dotti MT, et al. CSF biomarkers profile in CADASIL-a model of pure vascular dementia: usefulness in differential diagnosis in the dementia disorder. Int J Alzheimers Dis. 2010;2010:959257. 
31. Janelidze S, Stomrud E, Smith R, Palmqvist S, Mattsson N, et al. Cerebrospinal fluid p-tau217 performs better than p-tau181 as a biomarker of Alzheimer's disease. Nat Commun 2020;11:1683.

32. Bourbouli M, Rentzos M, Bougea A, Zouvelou V, Constantinides VC, et al. Cerebrospinal fluid TAR DNA-binding protein 43 combined with tau proteins as a candidate biomarker for amyotrophic lateral sclerosis and frontotemporal dementia spectrum disorders. Dement Geriatr Cogn Disord 2017;44:144-52.

33. Paraskevas GP, Bourbouli M, Zaganas I, Kapaki E. The emerging TDP-43 proteinopathy. Neuroimmunol Neuroinflammation $2018 ; 5: 17$.

34. Kapaki E, Paraskevas GP, Emmanouilidou E, Vekrellis K. The diagnostic value of CSF alpha-synuclein in the differential diagnosis of dementia with Lewy bodies vs. normal subjects and patients with Alzheimer's disease. PLoS One 2013;8:e81654.

35. Bougea A, Stefanis L, Paraskevas GP, Emmanouilidou E, Efthymiopoulou E, et al. Neuropsychiatric symptoms and $\alpha$-Synuclein profile of patients with Parkinson's disease dementia, dementia with Lewy bodies and Alzheimer's disease. J Neurol 2018;265:2295-301.

36. Janelidze S, Mattsson N, Palmqvist S, Smith R, Beach TG, et al. Plasma P-tau181 in Alzheimer's disease: relationship to other biomarkers, differential diagnosis, neuropathology and longitudinal progression to Alzheimer's dementia. Nat Med 2020;26:379-86.

37. Schindler SE, Bollinger JG, Ovod V, Mawuenyega KG, Li Y, et al. High-precision plasma $\beta$-amyloid 42/40 predicts current and future brain amyloidosis. Neurology 2019;93:e1647-59.

38. Wallin A, Kapaki E, Boban M, Engelborghs S, Hermann DM, et al. Biochemical markers in vascular cognitive impairment associated with subcortical small vessel disease - A consensus report. BMC Neurol 2017;17:102.

39. Rentzos M, Paraskevas GP, Kapaki E, Nikolaou C, Zoga M, et al. Circulating interleukin-15 in dementia disorders. J Neuropsychiatry Clin Neurosci 2007;19:318-25.

40. Rentzos M, Zoga M, Paraskevas GP, Kapaki E, Rombos A, et al. IL-15 is elevated in cerebrospinal fluid of patients with Alzheimer's disease and frontotemporal dementia. J Geriatr Psychiatry Neurol 2006;19:114-7.

41. Rentzos M, Paraskevas GP, Kapaki E, Nikolaou C, Zoga M, et al. Interleukin-12 is reduced in cerebrospinal fluid of patients with Alzheimer's disease and frontotemporal dementia. J Neurol Sci 2006;249:110-4. 